J. DIFFERENTIAL GEOMETRY

87 (2011) 493-514

\title{
RIGID AND NON-SMOOTHABLE SCHUBERT CLASSES
}

\author{
Izzet Coskun
}

\begin{abstract}
A Schubert class $\sigma_{\lambda}$ in the Grassmannian $G(k, n)$ is rigid if the only proper subvarieties representing that class are Schubert varieties $\Sigma_{\lambda}$. We prove that a Schubert class $\sigma_{\lambda}$ is rigid if and only if it is defined by a partition $\lambda$ satisfying a simple numerical criterion. If $\lambda$ fails this criterion, then there is a corresponding hyperplane class in another Grassmannian $G\left(k^{\prime}, n^{\prime}\right)$ such that the deformations of the hyperplane in $G\left(k^{\prime}, n^{\prime}\right)$ yield non-trivial deformations of the Schubert variety $\Sigma_{\lambda}$. We also prove that, if a partition $\lambda$ contains a sub-partition defining a rigid and singular Schubert class in another Grassmannian $G\left(k^{\prime}, n^{\prime}\right)$, then there does not exist a smooth subvariety of $G(k, n)$ representing $\sigma_{\lambda}$.
\end{abstract}

\section{Introduction}

A Schubert variety in the Grassmannian $G(k, n)$ is smooth if and only if it is a linearly embedded sub-Grassmannian $([\mathbf{L S}])$. Even when a Schubert variety is singular, there may be smooth subvarieties of $G(k, n)$ representing the same cohomology class. For example, the Schubert variety representing $\sigma_{1}$ in $G(2,5)$ is a singular hyperplane section of $G(2,5)$ in the Plücker embedding. However, Bertini's Theorem $([\mathbf{H}]$, II.8.18) guarantees that a general hyperplane section of $G(2,5)$ is a smooth variety representing $\sigma_{1}$. In contrast, $\sigma_{2}$ cannot be represented by a smooth subvariety of $G(2,5)$. In fact, any proper subvariety of $G(2,5)$ with cohomology class $\sigma_{2}$ is a Schubert variety. Nevertheless, there are many Schubert classes, such as $\sigma_{3,2,0}$ in $G(3,7)$, that admit non-trivial deformations but cannot be represented by a smooth, proper subvariety of $G(k, n)$.

In this paper, we characterize the rigid Schubert classes in Grassmannians. We also give a nearly-sharp criterion for determining when a Schubert class cannot be represented by a smooth subvariety of the Grassmannian. Throughout the paper we work over the field of complex numbers $\mathbb{C}$. We first introduce the necessary notation and state our results.

Received 10/22/2009. 
Schubert varieties. Let $V$ be an $n$-dimensional vector space and let $\lambda$ denote a partition with $k$ parts satisfying

$$
n-k \geq \lambda_{1} \geq \lambda_{2} \geq \cdots \geq \lambda_{k} \geq 0 .
$$

It is often convenient to group the repeated parts of $\lambda$ and write $\lambda=$ $\left(\mu_{1}^{i_{1}}, \ldots, \mu_{j}^{i_{j}}\right)$, where the parts $n-k \geq \mu_{1}>\mu_{2}>\cdots>\mu_{j} \geq 0$ of $\lambda$ occur $i_{1}, \ldots, i_{j}$ times (with $\sum_{s=1}^{j} i_{s}=k$ ), respectively. We will interchangeably use both notations.

Let $F_{\bullet}$ be a full flag in $V$. The Schubert variety $\Sigma_{\lambda}\left(F_{\bullet}\right)$ is the subvariety of $G(k, n)$ parameterizing $k$-dimensional linear spaces $W$ of $V$ such that

$$
\operatorname{dim}\left(W \cap F_{n-k+i-\lambda_{i}}\right) \geq i, \text { for } 1 \leq i \leq k .
$$

Let $\sigma_{\lambda}$ denote the cohomology class of $\Sigma_{\lambda}\left(F_{\bullet}\right)$. Schubert classes generate the extremal rays of the cone of effective classes and give an additive $\mathbb{Z}$ basis for the cohomology of $G(k, n)$. We say that a variety $X$ represents a cohomology class $c$ if $X$ is proper and the cohomology class of $X$ is $c$.

Definition 1.1. A Schubert class $\sigma_{\lambda}$ is called rigid if the only proper subvarieties of $G(k, n)$ representing $\sigma_{\lambda}$ are Schubert varieties.

Definition 1.2 (Rigid partition). We will call a partition $\lambda=$ $\left(\mu_{1}^{i_{1}}, \ldots, \mu_{j}^{i_{j}}\right)$ a rigid partition for $G(k, n)$ if there does not exist an index $1 \leq s<j$ with $i_{s}=1$ and $n-k>\mu_{s}=\mu_{s+1}+1$.

For example, $(4,4,2,0),(3,3,2,2),(4,3,1,1)$, and $(2,2,2,0)$ are rigid partitions for $G(4,8)$, whereas $(3,2,0,0),(4,4,1,0)$ are not rigid partitions for $G(4,8)$.

In recent years, the Schur rigidity of Schubert varieties has been extensively studied by differential geometers (see $[\mathbf{B r}],[\mathbf{H o 1}],[\mathbf{H o 2}]$, and $[\mathbf{W}])$. Recall that a Schubert class is Schur rigid if the only integral varieties of the corresponding Schur differential system (see Definition 40 of $[\mathbf{W}]$ or 2.8 .1 of $[\mathbf{B r}])$ are Schubert varieties. In [Ho2], Hong proves that a large class of Schubert varieties are Schur rigid. In this paper, we develop the algebro-geometric theory of rigidity and make no use of Schur rigidity. Our first theorem completely characterizes rigid Schubert classes.

Theorem 1.3. A Schubert class $\sigma_{\lambda}$ in $G(k, n)$ is rigid if and only if $\lambda$ is a rigid partition for $G(k, n)$.

EXAMPLE 1.4. Theorem 1.3 implies that the Schubert class $\sigma_{3,2,0}$ in $G(3,7)$ can be represented by proper subvarieties of $G(3,7)$ that are not Schubert varieties. A deformation of the Schubert variety $\Sigma_{3,2,0}$ can be obtained as follows. Fix a four-dimensional subspace $V^{\prime}$ of $V$. Let $Y$ be a general hyperplane section of the Grassmannian $G\left(2, V^{\prime}\right)$ in its Plücker embedding. Let $X$ be the subvariety of $G(3, V)$ parameterizing three-dimensional linear subspaces of $V$ that contain a two-dimensional 
subspace parameterized by $Y$. Then $X$ represents the class $\sigma_{3,2,0}$ in $G(3,7)$, but is not isomorphic to a Schubert variety (for instance, the singular locus of $X$ is irreducible, whereas the singular locus of $\Sigma_{3,2,0}$ has two irreducible components). Nevertheless, the class $\sigma_{3,2,0}$ cannot be represented by a smooth subvariety of $G(3,7)$. This example raises the problem of characterizing Schubert classes that can be represented by smooth subvarieties of $G(k, n)$.

Definition 1.5 (Non-smoothable partition). We will call a partition $\lambda=\left(\mu_{1}^{i_{1}}, \ldots, \mu_{j}^{i_{j}}\right)$ a non-smoothable partition for $G(k, n)$ if either there exists an index $1 \leq s<j$ such that $i_{s} \neq 1$ and $n-k>\mu_{s}$, or there exists an index $1 \leq s<j$ such that $n-k>\mu_{s} \neq \mu_{s+1}+1$.

For example, $(3,3,1,0),(3,2,2,1),(4,2,0,0)$ are non-smoothable partitions for $G(4,8)$, whereas $(3,2,1,0),(2,1,0,0),(4,4,2,1)$ are not nonsmoothable partitions for $G(4,8)$.

Theorem 1.6. Let $\lambda$ be a non-smoothable partition for $G(k, n)$. Then $\sigma_{\lambda}$ cannot be represented by a smooth subvariety of $G(k, n)$.

Theorem 1.6 is nearly sharp in a sense that we now make precise. Suppose $V^{\prime} \subset V$ is a subspace of dimension $m$. If $s \geq \max (0, k+m-$ $n)$, then the Grassmannian $G(s, m)$ can be embedded into $G(k, n)$ as follows. Let $U$ be a subspace of $V$ of dimension $k-s$ such that $U \cap V^{\prime}=0$. Given $\left[W^{\prime}\right] \in G(s, m)$, the span of $W^{\prime}$ and $U$ is a $k$-dimensional subspace of $V$. This induces an embedding of $G(s, m)$ into $G(k, n)$ compatible with the Plücker embedding. We will call a sub-Grassmannian of $G(k, n)$ obtained as the image of such a morphism a linearly embedded subGrassmannian. The Gysin image of the fundamental class of $G(s, m)$ is $\sigma_{\lambda}$, where

$$
\lambda=\left((n-k)^{k-s},(n-m+s-k)^{s}\right),
$$

and the Gysin image of the fundamental class of a hyperplane section in $G(s, m)$ is $\sigma_{\lambda^{\prime}}$, where

$$
\lambda^{\prime}=\left((n-k)^{k-s}, n-m+s-k+1,(n-m+s-k)^{s-1}\right)
$$

in the cohomology of $G(k, n)$. In particular, note that the Schubert classes $\sigma_{\lambda}$ with

$$
\lambda=\left((n-k)^{k-s}, p^{s}\right) \quad \text { or } \quad\left((n-k)^{k-s-1}, p+1, p^{s}\right),
$$

can be represented by smooth subvarieties of the Grassmannian $G(k, n)$ for any $0 \leq s \leq k$ and $0 \leq p \leq n-k-1$.

More generally, the space of Schubert varieties of class $\sigma_{n-r, n-r}$ contained in $G(2, n)$ is parameterized by the Grassmannian $G(r, n)$. Consider the subvariety of $G(r, n)$ parameterizing the locus of subvarieties of $G(2, n)$ with class $\sigma_{n-r, n-r}$ that are contained in a general hyperplane 
section of $G(2, n)$ in the Plücker embedding. Consider the incidence correspondence

$$
\begin{aligned}
I:=\{(X, H) \mid X & \left.\subset G(2, n) \cap H \text { with }[X]=\sigma_{n-r, n-r}\right\} \\
& \subset G(r, n) \times\left(\mathbb{P}_{\left(\begin{array}{l}
n \\
2
\end{array}\right)-1}\right)^{*}
\end{aligned}
$$

parameterizing pairs of a Schubert subvariety of $G(2, n)$ with class $\sigma_{n-r, n-r}$ and a hyperplane section of $G(2, n)$ in the Plücker embedding containing it. The first projection exhibits $I$ as a projective space bundle over $G(r, n)$ with fibers isomorphic to projective spaces of dimension $\left(\begin{array}{l}n \\ 2\end{array}\right)-\left(\begin{array}{l}r \\ 2\end{array}\right)-1$. Consequently, $I$ is smooth. By generic smoothness $([\mathbf{H}]$, III.10.7), a general fiber of the second projection is a smooth subvariety of $G(r, n)$. This variety is the zero locus of a section of the vector bundle $\bigwedge^{2} S^{*}$ over $G(r, n)$, where $S$ denotes the tautological bundle of $G(r, n)$, amd hence has cohomology class $\sigma_{r-1, r-2, r-3, \ldots, 3,2,1,0}$. Using the construction in the previous paragraph, it follows that the Schubert classes $\sigma_{\lambda}$ in $G(k, n)$ with

$$
\lambda=\left((n-k)^{s}, p, p-1, p-2, \ldots, p-k-s+2, p-k+s+1\right)
$$

with $k \geq s \geq 0$ and $p-k+s+1 \geq 0$ can be represented by smooth subvarieties of $G(k, n)$. Consequently, Theorem 1.6 is sharp in the following sense. Let $\lambda$ be a partition with $i_{t}=1$ and $\mu_{t}=\mu_{t+1}+1$ for all $1 \leq t<j$; then $\sigma_{\lambda}$ can be represented by a smooth subvariety of $G\left(k, k+3+\mu_{j}\right)$. However, this still leaves the following problem open.

Problem 1.7. Let $\lambda=\left(\mu_{1}^{i_{1}}, \ldots, \mu_{j}^{i_{j}}\right)$ be a partition with $i_{t}=1$ and $\mu_{t}=\mu_{t+1}+1$ for every $1 \leq t<j$ and $\sum_{t=1}^{j} i_{t}=k$. For which $n$ does there exist a smooth variety in $G(k, n)$ representing the class $\sigma_{\lambda}$ ?

Let us explain the strategy of the proof of Theorem 1.6 in the case of $G(2, n)$. Theorem 1.6 asserts that the only classes $\sigma_{a, b}$ that can be represented by smooth subvarieties of $G(2, n)$ have either $a=n-2$ or $a-b \leq 1$. Let $\sigma_{a, b}$ be a Schubert class in $G(2, n)$ such that $a<n-2$ and $a-b>1$. We need to show that $\sigma_{a, b}$ cannot be represented by a smooth subvariety of $G(2, n)$. Suppose to the contrary that $X$ is a smooth, proper subvariety of $G(2, n)$ representing $\sigma_{a, b}$. Then by intersecting $X$ with a general Schubert variety $\Sigma_{n-3-a, n-3-a}$ (which is smooth), we obtain a smooth, proper variety that represents the class $\sigma_{n-3, n-3+b-a}$. Hence, to prove Theorem 1.6 for $G(2, n)$, it suffices to show that a variety $Y$ representing the class $\sigma_{n-3, n-3-c}$ for $c>1$ cannot be smooth.

The dimension of $Y$ is $c+2$. By Pieri's formula $([\mathbf{F}], 14.6 .1)$, the degree of $Y$ in the Plücker embedding of $G(2, n)$ is $c+1$. Any subvariety of $G(2, n)$ representing a Schubert class $\sigma_{\lambda}$ can be specialized to a scheme whose support is a Schubert variety $\Sigma_{\lambda}$. In particular, the dimension of the linear span of $Y$ in the Plücker embedding has to be greater than or equal to $2 c+2$, the dimension of the linear span of a Schubert variety 
$\Sigma_{n-3, n-3-c}$. We conclude that the Plücker embedding of $Y$ has to be a minimal variety in projective space of dimension $c+2$ and degree $c+1$.

Recall that the degree $d$ of an $n$-dimensional, non-degenerate, irreducible variety in $\mathbb{P}^{r}$ is bounded by $d \geq r+1-n$. The varieties for which equality holds are called minimal varieties and have been classified by Del Pezzo $[\mathbf{D}]$ and Bertini $[\mathbf{B e}]$. A modern proof can be found in $[\mathbf{E H}]$. Here we recall the statement of the classification of varieties of minimal degree for the convenience of the reader:

Classification of varieties of minimal degree. [EH, theorem 1] If $X$ is an irreducible, non-degenerate variety of dimension $n$ and degree $r+1-n$ in $\mathbb{P}^{r}$, then $X$ is either a quadric hypersurface, a rational normal scroll, the Veronese surface in $\mathbb{P}^{5}$, or a cone over any of these varieties.

Since $c>1$, the degree of $Y$ is at least three. The dimension of a smooth rational scroll or a Veronese surface is less than or equal to its degree. Since the degree of $Y$ is less than its dimension, by the classification of the varieties of minimal degree, $Y$ is a cone. Therefore, $Y$ is singular. In fact, it is easy to see that $Y$ is a cone over a rational normal scroll. This suffices to prove Theorem 1.6 for $G(2, n)$. The proof in the general case follows the same strategy; however, the base cases that need to be treated are not necessarily minimal varieties. Fortunately, the base cases are all cones over Segre varieties and do not admit any non-trivial infinitesimal deformations.

Theorem 1.3 is then proved by analyzing the singular locus of a variety representing a Schubert class $\sigma_{\lambda}$. For example, any proper variety $X$ representing the class $\sigma_{n-3,0}$ in $G(2, n)$ has to be a cone over a rational normal scroll. The cone point $p$ of $X$ determines a two-dimensional subspace $L$ of $V$. Since the Zariski tangent space to $X$ at $p$ has dimension $2 n-4$, we conclude that the span of $X$ has to be the projectivized tangent space to $G(2, n)$ at $p$. Hence, $X$ is contained in the Schubert variety of two-dimensional subspaces of $V$ that intersect $L$. Since both varieties are irreducible and of the same dimension, $X$ is equal to the Schubert variety. The proof in the general case proceeds by showing that the singular locus determines the partial flag defining the Schubert variety of the same class.

There are many variants and generalizations of the problems we study in this paper.

Problem 1.8. Which non-negative linear combinations of Schubert classes in $G(k, n)$ can be represented by irreducible/smooth proper subvarieties?

Hong's results in [Ho2] imply that positive, integral multiples of many Schubert classes cannot be represented by smooth, proper subvarieties of the Grassmannian. On the other hand, it is possible to find 
many linear combinations that can be represented by smooth subvarieties by simply exhibiting smooth subvarieties of the Grassmannian. The following proposition gives some simple constructions of smooth subvarieties of Grassmannians. We thus obtain a large collection of cohomology classes that can be represented by smooth subvarieties.

Proposition 1.9. The following are examples of classes that can be represented by smooth subvarieties of the Grassmannian.

1) (Bertini) The cohomology class $r \sigma_{1}^{m}$ can be represented by a smooth subvariety of $G(k, n)$ for every $r>0$ and $0<m<k(n-k)$.

2) The cohomology class $\sum_{\nu} \alpha_{\lambda, \mu}^{\nu} \sigma_{\nu}$, where $\alpha_{\lambda, \mu}^{\nu}$ is the LittlewoodRichardson coefficient, can be represented by a smooth subvariety of $G(k, n)$ provided that $\lambda$ and $\mu$ have the form $\left(b_{1}^{i_{1}}, \ldots, b_{j}^{i_{j}}\right)$ and $\left(c_{1}^{i_{j}}, \ldots, c_{j}^{i_{1}}\right)$, respectively, with the property that $b_{i}+c_{j-i} \geq n-k$.

3) The cohomology classes $r \sigma_{\left((n-k)^{k-1}, a\right)}$, with $r>0$ and $a<n-$ $k$, and $r \sigma_{\left((n-k)^{s},(n-k-1)^{k-s}\right)}$, with $r>0$ and $k>s>0$, can be represented by smooth subvarieties of $G(k, n)$.

4) The space of projective linear spaces of dimension $k$ contained in a general complete intersection in $\mathbb{P}^{n}$ is either empty or a smooth subvariety in $G(k+1, n+1)$. Irreducible components of the spaces of linear spaces contained in Grassmannians are smooth.

5) Let $Y_{i} \in G\left(k_{i}, n_{i}\right), 1 \leq i \leq j$, be smooth subvarieties with classes $c_{i}=\sum c_{\lambda}^{i} \sigma_{\lambda}$. Let $\tilde{c}_{i}=\sum c_{\lambda}^{i} \sigma_{\tilde{\lambda}}$, where $\tilde{\lambda}$ is the partition obtained by adding $n-n_{i}-k+k_{i}$ to each of the parts of $\lambda$ and a tail of $k-k_{i}$ zeros. Then, the product $Y_{1} \times \cdots \times Y_{j}$ can be embedded as a smooth subvariety of $G\left(\sum_{i=1}^{j} k_{i}, \sum_{i=1}^{j} n_{i}\right)$ representing the class $\prod \tilde{c}_{i}$.

The following problem is a closely related variant of Problem 1.8:

Problem 1.10. Which cohomology classes in the Grassmannian can be expressed as a linear combination (allowing negative signs!) of the classes of smooth proper subvarieties?

EXAMPLE 1.11. While $\sigma_{2}$ in $G(2,5)$ cannot be represented by a smooth, proper subvariety, it can be expressed as the difference of the classes of two smooth subvarieties:

$$
\sigma_{2}=\left(\sigma_{1}\right)^{2}-\sigma_{1,1}
$$

The Schubert variety $\Sigma_{1,1}$ is smooth and by Bertini's Theorem a general codimension two linear section of the Plücker embedding of $G(2,5)$ is a smooth representative of $\left(\sigma_{1}\right)^{2}$. In $[\mathbf{K} \mathbf{1}]$, Kleiman proves that if $c$ is the cohomology class of a variety of dimension $d \leq \frac{k(n-k)}{2}$ in $G(k, n)$, then $q$ ! c, where $q=k(n-k)-d-1$, can be written as a linear combination of classes of smooth subvarieties. In $[\mathbf{K L}]$, Kleiman and Landolfi 
conjectured that in $G(3,6)$ the codimension two Schubert cycles cannot be deformed into smooth cycles by rational equivalence. In [HRT], R. Hartshorne, E. Rees, and E. Thomas have shown the stronger fact that $\sigma_{2}$ in $G(3,6)$ cannot even be written as a linear combination of the classes of smooth closed submanifolds of $G(3,6)$. The complete characterization of classes that can be expressed as a linear combination of smooth subvarieties currently seems out of reach.

Acknowledgments. It is a pleasure to thank Robert Bryant and Joe Harris for many enlightening conversations. The author would also like to thank Sara Billey, Anders Buch, Lawrence Ein, Bill Fulton, Brendan Hassett, János Kollár, Fumei Lam, Jason Starr, Ravi Vakil, and the anonymous referees. I am indebted to the Mathematical Sciences Research Institute for providing a wonderful work environment where part of this project was completed.

During the preparation of this article, the author was partially supported by the NSF grant DMS-0737581, the NSF CAREER grant DMS0950951535, and an Alfred P. Sloan Foundation Fellowship.

\section{Singularities of Schubert Varieties}

In this section, we recall some facts about the singularities of Schubert varieties in Grassmannians used in the sequel. There is a wealth of information about the singularities of Schubert varieties: Schubert varieties are normal, Cohen-Macaulay with rational singularities and admit natural resolutions such as the Bott-Samelson resolution. The singular loci of Schubert varieties and the multiplicity along each singular locus can be explicitly described. We refer the reader to the excellent book by Billey and Lakshmibai for further information, detailed history, and extensive references $[\mathbf{B L}]$.

The Bott-Samelson/Zelevinsky Resolution. Schubert varieties in the Grassmannian admit a natural resolution $f: \tilde{\Sigma} \rightarrow \Sigma$ such that the exceptional locus of $f$ has codimension at least two. Let $\lambda=\left(\mu_{1}^{i_{1}}, \mu_{2}^{i_{2}}, \ldots, \mu_{j}^{i_{j}}\right)$ be a partition for $G(k, n)$ and let $a_{s}=\sum_{l=1}^{s} i_{l}$. Let $\tilde{\Sigma}_{\lambda}$ be the Schubert variety in the flag variety $F\left(a_{1}, a_{2}, \ldots, a_{j} ; n\right)$ defined by

$\tilde{\Sigma}_{\lambda}:=\left\{\left(V_{1}, \ldots, V_{j}\right) \in F\left(a_{1}, a_{2}, \ldots, a_{j} ; n\right) \mid V_{s} \subset F_{n-k+a_{s}-\lambda_{a_{s}}}, 1 \leq s \leq j\right\}$.

Since $\tilde{\Sigma}_{\lambda}$ is an iterated tower of Grassmannian bundles, it is smooth. Furthermore, $\tilde{\Sigma}_{\lambda}$ maps onto $\Sigma_{\lambda}$ by the natural projection

$$
\pi: F\left(a_{1}, a_{2}, \ldots, a_{j} ; n\right) \rightarrow G(k, n) .
$$

By Zariski's Main Theorem, the map is an isomorphism over the locus of $k$-planes $W$ parameterized by $\Sigma_{\lambda}$ with the property that $\operatorname{dim}(W \cap$ 
$\left.F_{n-k+a_{s}-\lambda_{a_{s}}}\right)=a_{s}$ for $1 \leq s \leq j$. The map $\left.\pi\right|_{\tilde{\Sigma}_{\lambda}}$ has positive dimensional fibers over the locus of $k$-planes $W$ with the property that $\operatorname{dim}\left(W \cap F_{n-k+a_{s}-\lambda_{a_{s}}}\right)>a_{s}$ for some $1 \leq s \leq j$.

Definition 2.1. Given a partition $\lambda=\left(\mu_{1}^{i_{1}}, \ldots, \mu_{j}^{i_{j}}\right)$ for $G(k, n)$, a singular partition $\lambda_{s}$ associated to $\lambda$ is $\lambda_{s}=\left(\mu_{1}^{i_{1}}, \ldots,\left(\mu_{s}+1\right)^{i_{s}+1}\right.$, $\left.\mu_{s+1}^{i_{s+1}-1}, \ldots, \mu_{j}^{i_{j}}\right)$ for some $1 \leq s<j$, provided that the resulting partition is admissible for $G(k, n)$.

REMARK 2.2. In terms of Young diagrams, the singular partitions are obtained from $\lambda$ by adding a hook to $\lambda$ so that the resulting partition is still admissible for $G(k, n)$. See Figure 1 for an example.

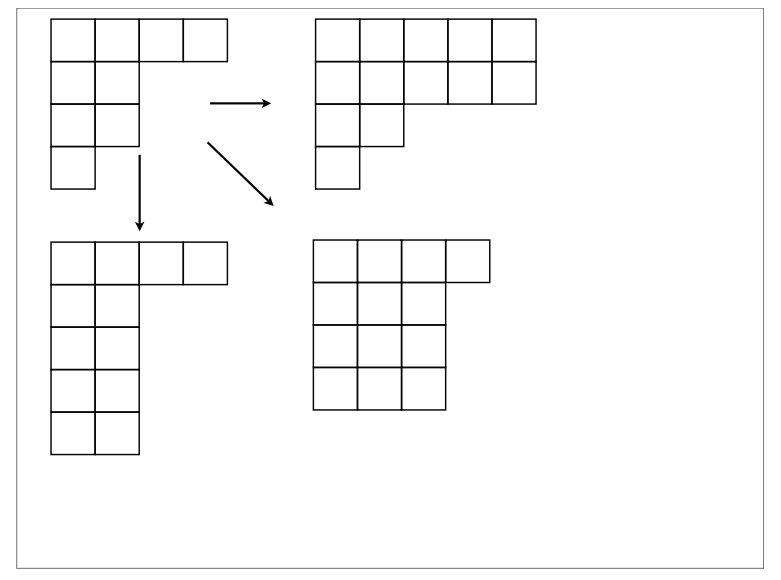

Figure 1. The singular partitions associated to $(4,2,2,1,0)$ are $(5,5,2,1),(4,2,2,2,2)$, and $(4,3,3,3)$.

The exceptional locus of the map $\left.\pi\right|_{\tilde{\Sigma}_{\lambda}}$ consists of the union of the inverse images of $\Sigma_{\lambda_{s}}\left(F_{\bullet}\right)$ for every singular partition associated to $\lambda$. The locus $\pi^{-1}\left(\Sigma_{\lambda_{s}}\left(F_{\bullet}\right)\right)$ is irreducible and has codimension greater than one. $\left(\Sigma_{\lambda_{s}}\right.$ has codimension $\mu_{s}-\mu_{s+1}+i_{s}+1$ in $\Sigma_{\lambda}\left(F_{\bullet}\right)$ and the general fiber dimension of $\pi$ restricted to $\pi^{-1}\left(\Sigma_{\lambda_{s}}\left(F_{\bullet}\right)\right)$ is $i_{s}$.) Furthermore, the exceptional locus of $\pi$ is empty if and only if $j=1$ or $j=2$ and $\mu_{1}=n-k$. The following lemma allows us to determine the singular locus of a Schubert variety.

Lemma 2.3. Let $f: X \rightarrow Y$ be a birational morphism from a smooth, projective variety $X$ onto a normal projective variety $Y$. Assume that $f$ is an isomorphism in codimension one. Then $p \in Y$ is a singular point if and only if $f^{-1}(p)$ is positive dimensional.

Proof. By Zariski's Connectedness Theorem ([H], III.11.4), if $f^{-1}(p)$ is not positive dimensional, then it is a point. Consider the open set 
$U$ in $Y$ consisting of the locus where $f^{-1}(p)$ is a single point. Then, by Zariski's Main Theorem, $\left.f\right|_{f^{-1}(U)}: f^{-1}(U) \rightarrow U$ is an isomorphism. Since $f^{-1}(U)$ is a Zariski open subset of a smooth variety, $f^{-1}(U)$ and consequently $U$ is smooth. Conversely, suppose $p$ is a point such that $f^{-1}(p)$ is positive dimensional. If $p$ is smooth, then to check that the map $f$ is a local isomorphism, it suffices to check that the Jacobian does not vanish. Since the map $f$ is an isomorphism in codimension one and the vanishing locus of the Jacobian of $f$ is a divisor, we conclude that the Jacobian does not vanish. On the other hand, since $f$ is not a local isomorphism around $f^{-1}(p)$, we conclude that $p$ has to be a singular point.

q.e.d.

We obtain the following two corollaries due to Lakshmibai and Seshadri ([LS]).

Corollary 2.4 ([LS]). A Schubert variety $\Sigma_{\lambda}$ in $G(k, n)$ is smooth if and only if it is a linearly embedded sub-Grassmannian, or equivalently, $\lambda=\left((n-k)^{s}, p^{k-s}\right)$.

Corollary $2.5([\mathbf{L S}])$. The singular locus of a Schubert variety $\Sigma_{\lambda}\left(F_{\bullet}\right)$ in $G(k, n)$ is the union of all the Schubert varieties $\Sigma_{\lambda_{s}}\left(F_{\bullet}\right)$, where $\lambda_{s}$ is a singular partition associated to $\lambda$.

OBSERVATION 2.6. In particular, by Kleiman's Transversality Theorem $([\mathbf{K 2}])$, the intersection of a collection of general Schubert varieties $\Sigma_{\lambda^{1}}, \ldots, \Sigma_{\lambda^{j}}$ is smooth and non-empty provided that $\sigma_{\lambda^{1}} \cdots \sigma_{\lambda^{j}} \neq 0$ and $\sigma_{\lambda_{s}^{i}} \cdot \prod_{l \neq i} \sigma_{\lambda^{l}}=0$ for every $1 \leq i \leq j$ and every singular partition $\lambda_{s}^{i}$ associated to $\lambda^{i}$.

Using this Observation 2.6, we can prove Proposition 1.9.

Proof of Proposition 1.9. The class $r \sigma_{1}$, with $r>0$, is represented by the intersection of $G(k, n)$ in its Plücker embedding with a hypersurface of degree $r$. Hence, $r \sigma_{1}^{m}$ is represented by the intersection of $G(k, n)$ with a general hypersurface of degree $r$ and $m-1$ general hyperplanes. By Bertini's Theorem ([H], II.8.18), this intersection is smooth. This proves (1).

(2) is a special case of Observation 2.6. Let $a_{s}=\sum_{l=1}^{s} i_{l}$. Let $F_{\bullet}$ and $G_{\bullet}$ be two general flags defining $\Sigma_{\lambda}$ and $\Sigma_{\mu}$, respectively. If $b_{i}+c_{j-i} \geq$ $n-k$, then $F_{n-k+a_{i}-b_{i}} \cap G_{n-a_{i}-c_{j-i}}=\{0\}$ for every $i$. Consequently, the intersection of the two Schubert varieties $\Sigma_{\lambda} \cap \Sigma_{\mu}$ is isomorphic to the product of Grassmannians $\prod_{l} G\left(i_{l}, n_{l}\right)$, where $n_{l}=n-k+i_{l}-b_{l}-c_{j-l+1}$ for $1 \leq l \leq j$. Hence, this intersection is smooth.

The Schubert cycles $\sigma_{(n-k)^{k-1}, 0}$ and $\sigma_{(n-k-1)^{k}}$ are projective spaces $\mathbb{P}^{n-k}$ and $\mathbb{P}^{k}$, respectively. Smooth complete intersections of type $\left(r, 1^{a-1}\right)$ and $\left(r, 1^{s-1}\right)$ in these projective spaces have cohomology classes $r \sigma_{(n-k)^{k-1}, a}$ and $r \sigma_{(n-k)^{s},(n-k-1)^{k-s}}$, respectively. This proves (3). 
The incidence correspondence $I:=\{(L, X) \mid L \subset X\}$, where $L$ is a linear space and $X$ is a complete intersection of degrees $d_{1}, \ldots, d_{j}$, is smooth. By generic smoothness $([\mathbf{H}]$, III.10.7), the general fiber of the second projection is empty or smooth. This proves the first part of (4). The second part of (4) is well-known.

Let $V_{i}$ of dimension $n_{i}$ be $j$ general linear spaces in an $n=\sum_{i=1}^{j} n_{i}$ dimensional linear space. Since $V_{i}$ are general, the span of any $j-1$ of them intersects the last one trivially. Given varieties $Y_{i} \subset G\left(k_{i} V_{i}\right)$, consider the following variety:

$$
Y=\left\{[W] \in G(k, n) \mid\left[W \cap V_{i}\right] \in Y_{i}\right\} .
$$

Then $Y$ is isomorphic to $\prod_{i=1}^{j} Y_{i}$. Given a point $\left(W_{1}, \ldots, W_{j}\right) \in \prod_{i=1}^{j} Y_{i}$, the span $\overline{W_{1} \cdots W_{j}}$ is a $k$-dimensional subspace in $Y$. Conversely, for $W \in Y,\left(W \cap V_{1}, \ldots, W \cap V_{j}\right) \in \prod_{i=1}^{j} Y_{i}$. It is clear that these maps are inverses of each other. The statement about the cohomology classes is straightforward. This completes the proof of Proposition 1.9. $\quad$ q.e.d.

\section{Rigidity of Smooth Schubert Varieties}

In this section, we prove a proposition that will allow us to reduce Theorem 1.6 to a few basic cases. An immediate corollary of the proposition is the rigidity of smooth Schubert varieties.

Proposition 3.1. Let $\lambda$ be a partition for $G(k, n)$ such that $\lambda_{1}=$ $\cdots=\lambda_{s}=n-k$ and $\lambda_{k}=r$. Let $X$ be a subvariety of $G(k, n)$ representing the cohomology class $\sigma_{\lambda}$. Then the linear spaces parameterized by $X$ contain a fixed $s$-dimensional linear space and are contained in a fixed $(n-r)$-dimensional linear space.

Proof. For the proof of this proposition, it is more convenient to think of the Grassmannian $G(k, n)$ as the Grassmannian $\mathbb{G}(k-1, n-1)$ of projective $(k-1)$-dimensional linear spaces in $\mathbb{P} V$. Let $\lambda$ be a partition with $\lambda_{s}=n-k$ and $\lambda_{k}=r$. Let $X$ be a variety representing the class $\sigma_{\lambda}$. Consider the incidence correspondence

$$
I_{X}=\{(p,[W]) \mid[W] \in X, p \in W\} \subset \mathbb{P}^{n-1} \times \mathbb{G}(k-1, n-1) .
$$

Let $\pi_{1}$ and $\pi_{2}$ denote the two projections to $\mathbb{P}^{n-1}$ and $\mathbb{G}(k-1, n-$ $1)$, respectively. Since a Schubert class is indecomposable, $X$ is an irreducible variety. The fibers of $\pi_{2}$ are projective spaces of dimension $k-1$; hence $I_{X}$ is irreducible. Consequently, the first projection $\pi_{1}\left(I_{X}\right)$ is an irreducible, projective variety in $\mathbb{P}^{n-1}$.

Let $L \cong \mathbb{P}^{r-1}$ be a general linear space. The class of the locus in $\mathbb{G}(k-1, n-1)$ that parameterizes the set of $\mathbb{P}^{k-1}$ that intersect $L$ is the Pieri class $\sigma_{n-k-r+1}$. Since the cup product of this class with $\sigma_{\lambda}$ is zero, we conclude that a general linear space of dimension $r-1$ does not intersect $\pi_{1}\left(I_{X}\right)$. Therefore, $\operatorname{dim}\left(\pi_{1}\left(I_{X}\right)\right) \leq n-r-1$. On the other 
hand, by Pieri's formula ([F], 14.6.1), the cup product of $\sigma_{\lambda}$ with $\sigma_{n-k-r}$ is not zero; hence $\operatorname{dim}\left(\pi_{1}\left(I_{X}\right)\right)=n-r-1$. In order to conclude that the linear spaces parameterized by $X$ are contained in a fixed codimension $r$ linear space, it suffices to prove that the degree of $\pi_{1}\left(I_{X}\right)$ (with its reduced induced structure) is equal to one.

Let $\lambda^{*}$ denote the partition dual to $\lambda$ given by $\lambda_{i}^{*}=n-k-\lambda_{k-i+1}$. Since the cup product of $X$ with $\sigma_{\lambda^{*}}$ is equal to the Poincaré dual of the point class, by Kleiman's Transversality Theorem $([\mathbf{K} 2])$, a general Schubert variety representing $\sigma_{\lambda^{*}}$ intersects $X$ in a reduced point. Furthermore, since $\pi_{1}\left(I_{X}\right)$ is a variety of dimension $n-r-1$, a general projective linear space of dimension $r$ intersects $\pi_{1}\left(I_{X}\right)$ in finitely many reduced points.

Fix a general linear space $\mathbb{P} F_{r+1} \cong \mathbb{P}^{r}$ such that the intersection of $\mathbb{P} F_{r+1}$ with $\pi_{1}\left(I_{X}\right)$ consists of finitely many reduced points $q_{1}, \ldots, q_{j}$ and there exists a partial flag $F_{\bullet}$ beginning with $F_{r+1}$ such that the Schubert variety with class $\sigma_{\lambda^{*}}$ defined with respect to $F_{\bullet}$ intersects $X$ in a reduced point. By the transitivity of the monodromy group ( $[\mathbf{H M}]$, 6.45 ), we may assume that any geometric property that occurs at one of the intersection points $q_{i}$ also occurs at the others. By construction, there is a linear space $[\Lambda] \in X$ such that $[\Lambda]=X \cap \Sigma_{\lambda^{*}}\left(F_{\bullet}\right)$. We must necessarily have $\mathbb{P} \Lambda \cap \mathbb{P} F_{r+1}=q_{i}$ for one of the intersection points $q_{i} \in \pi_{1}\left(I_{X}\right) \cap \mathbb{P} F_{r+1}$. In particular, by the transitivity of the monodromy group, we may assume that for each $q_{i}$ in $\mathbb{P} F_{r+1} \cap \pi_{1}\left(I_{X}\right)$, there exists a linear space $\Lambda_{q_{i}}$ parameterized by $X$ and a Schubert cycle $\Sigma_{\lambda^{*}}\left(F_{\bullet}^{q_{i}}\right)$ defined with respect to a partial flag containing $F_{r+1}$ such that $X$ and $\Sigma_{\lambda^{*}}\left(F_{\bullet}^{q_{i}}\right)$ intersect transversely at $\left[\Lambda_{q_{i}}\right]$. Note that $\operatorname{dim}\left(\Lambda_{q_{i}} \cap F_{r+1}\right)=1$.

The locus of partial flags

$$
G_{\bullet}=F_{r+1}=G_{n-k+1-\lambda_{1}^{*}} \subset G_{n-k+2-\lambda_{2}^{*}} \cdots \subset G_{n-\lambda_{k}^{*}}
$$

starting with the fixed general linear space $F_{r+1}$ such that the Schubert variety $\Sigma_{\lambda^{*}}\left(G_{\bullet}\right)$ intersects $X$ transversely is a non-empty, Zariski-open subset $U$ of a partial flag variety. Since a non-empty Zariski open subset of an irreducible variety is connected, $U$ is connected. There is a natural morphism from $U$ to the points of intersection $\mathbb{P} F_{r+1} \cap \pi_{1}\left(I_{X}\right)$ sending a partial flag $G_{\bullet}$ to $\mathbb{P}\left(\Lambda \cap F_{r+1}\right)$, where $[\Lambda]=X \cap \Sigma_{\lambda^{*}}\left(G_{\bullet}\right)$. This map surjects onto $\mathbb{P} F_{r+1} \cap \pi_{1}\left(I_{X}\right)$. Since the image of a connected set is connected, we conclude that the intersection of $\pi_{1}\left(I_{X}\right)$ with $\mathbb{P} F_{r+1}$ is connected. Consequently, $\mathbb{P} F_{r+1} \cap \pi_{1}\left(I_{X}\right)$ is a single point. Therefore, $\pi_{1}\left(I_{X}\right)$ has degree one and is a linear space. This proves that the linear spaces parameterized by $X$ have to be contained in a fixed codimension $r$ linear space.

The fact that the linear spaces parameterized by $X$ contain a fixed $s$ dimensional linear space follows by duality. The Grassmannian $G(k, n)$ is isomorphic to the Grassmannian $G(n-k, n)$. Under this isomorphism, 
a Schubert class $\sigma_{\lambda}$ is replaced by $\sigma_{\lambda^{\prime}}$, where $\lambda^{\prime}$ is the partition given by the columns of the Young diagram associated to $\lambda$. In particular, $\lambda^{\prime}$ satisfies $\lambda_{n-k}^{\prime}=s$. By the previous paragraph, all the linear spaces parameterized by the image of $X$ under this isomorphism are contained in a fixed codimension $s$ linear space. Under the duality, this linear space produces a fixed linear space of dimension $s$ contained in all the linear spaces parameterized by $X$. This concludes the proof. q.e.d.

As a corollary, we recover a well-known rigidity result (see $[\mathbf{B r}]$, [Ho1], or [Ho2]).

Corollary 3.2. Any proper subvariety of $G(k, n)$ representing the Schubert class $\sigma_{\lambda}$ with $\lambda=\left((n-k)^{s}, p^{k-s}\right)$ is a Schubert variety. Consequently, the smooth Schubert varieties in Grassmannians are rigid.

Proof. Let $X$ be a variety representing the cohomology class $\sigma_{\lambda}$ where $\lambda=\left((n-k)^{s}, p^{k-s}\right)$. By Proposition 3.1, every linear space parameterized by $X$ contains a fixed linear space of dimension $s$ and is contained in a fixed linear space of codimension $p$. The dimension of $X$ is $(k-s) p$. Since the Schubert variety parameterizing $k$-dimensional linear spaces containing a fixed $s$-dimensional linear space and contained in a fixed codimension $p$ linear space is irreducible of dimension $(k-s) p$ and contains $X$, we conclude that $X$ must be equal to the Schubert variety. q.e.d.

REMARK 3.3. In $[\mathbf{B r}]$ and [Ho1], Bryant and Hong, using more involved differential geometric arguments, prove that smooth Schubert varieties other than non-maximal linear spaces in Grassmannians are Schur rigid.

Next we would like to define some operations on partitions that will allow us to state a reduction lemma.

Definition 3.4. Let $\lambda=\left(\lambda_{1}, \ldots, \lambda_{k}\right)$ be a partition for $G(k, n)$.

1) If $\lambda_{1}<n-k$, then define the partition $\lambda+\underline{1}$ to be the partition $\left(\lambda_{1}+1, \ldots, \lambda_{k}+1\right)$ for $G(k, n)$ obtained by adding one to each part.

2) If $\lambda_{k}=0$, define the partition $\lambda^{+}$to be the partition $\left(n-k, \lambda_{1}, \ldots\right.$, $\left.\lambda_{k-1}\right)$ for $G(k, n)$ obtained by omitting the part $\lambda_{k}=0$ and adding the part $n-k$ to the resulting partition.

3) If $\lambda_{1}=n-k$, define the partition $\lambda^{-}$to be the partition for $G(k-1, n-1)$ obtained by omitting the part $\lambda_{1}$ from $\lambda$.

Lemma 3.5 (Reduction Lemma). Let $\sigma_{\lambda}$ be a Schubert class in $G(k, n)$.

1) If $\lambda_{1}<n-k$ and $\sigma_{\lambda+\underline{1}}$ cannot be represented by a smooth, proper subvariety of $G(k, n)$, then $\sigma_{\lambda}$ cannot be represented by a smooth, proper subvariety of $G(k, n)$. 
2) If $\lambda_{k}=0$ and $\sigma_{\lambda^{+}}$cannot be represented by a smooth, proper subvariety of $G(k, n)$, then $\sigma_{\lambda}$ cannot be represented by a smooth, proper subvariety of $G(k, n)$.

3) If $\lambda_{1}=n-k$ and $\sigma_{\lambda^{-}}$cannot be represented by a smooth, proper subvariety of $G(k-1, n-1)$, then $\sigma_{\lambda}$ cannot be represented by a smooth, proper subvariety of $G(k, n)$.

Proof. Suppose that $\lambda_{1}<n-k$ and that $X$ is a smooth, proper subvariety representing $\sigma_{\lambda}$. By Kleiman's Transversality Theorem, the intersection of $X$ with a general smooth Schubert variety $\Sigma_{\left(1^{k}\right)}$ is a smooth, proper subvariety of $G(k, n)$. By the Littlewood-Richardson rule $([\mathbf{F}], 14.6 .2), X \cap \Sigma_{\left(1^{k}\right)}$ represents the cohomology class $\sigma_{\lambda+\underline{1}}$. This proves Part (1) of the lemma.

Suppose that $\lambda_{k}=0$ and that $X$ is a smooth, proper subvariety representing $\sigma_{\lambda}$. By Kleiman's Transversality Theorem, the intersection of $X$ with a general smooth Schubert variety $\Sigma_{\left(n-k, 0^{k-1}\right)}$ is a smooth, proper subvariety of $G(k, n)$. This variety represents the class $\sigma_{\lambda^{+}}$. This proves Part (2) of the lemma.

Finally, if $\lambda_{1}=n-k$, by Proposition 3.1, any variety $X$ representing the class $\sigma_{\lambda}$ is contained in a linearly embedded sub-Grassmannian $G(k-1, n-1)$. The class of $X$ in $G(k-1, n-1)$ is $\sigma_{\lambda^{-}}$. Part (3) of the lemma follows.

q.e.d.

\section{The Proofs of Theorem 1.3 and Theorem 1.6}

In this section we give the proofs of Theorem 1.3 and Theorem 1.6. The proofs of these theorems depend on a few base cases which we establish in a series of lemmas and propositions.

Lemma 4.1. Let $X$ be a proper variety representing a Schubert class $\sigma_{\lambda}$. Then there exists a flat, one-parameter family $\mathcal{Y} \rightarrow B$ over a smooth curve such that the general fiber is isomorphic to $X$ and the special fiber $\mathcal{Y}_{0}$ is isomorphic to a Schubert variety $\Sigma_{\lambda}$.

Proof. The Grassmannian $G(k, n)$ has a cell-decomposition by Schubert cells, where each cell is isomorphic to an affine space. Suppose that $X$ is contained in the closure of a cell. If $X$ is equal to the closure of the cell, then $X$ is a Schubert variety. Otherwise, by projecting from a point not on $X, X$ can be degenerated to a variety contained in a cell of lower dimension. Consequently, using the action of a one-parameter subgroup of $G L(n)$, every subvariety of $G(k, n)$ can be degenerated to a scheme supported on a union of Schubert varieties (usually with multiplicities). By the properness of the Hilbert scheme, a flat limit always exists. Since the class $\sigma_{\lambda}$ is indecomposable in cohomology, a variety $X$ representing $\sigma_{\lambda}$ is irreducible. Furthermore, the flat limit of the family is supported on a Schubert variety $\Sigma_{\lambda}$ and is generically reduced. Since the Schubert 
variety $\Sigma_{\lambda}$ is normal, Hironaka's Lemma $([\mathbf{H}]$, theorem III.9.11, $[\mathbf{K o}]$ theorem 2 or $[\mathbf{H i}]$ ) guarantees that there are no embedded components in the limit.

q.e.d.

Proposition 4.2. Let $\lambda=\left((n-k)^{r},(n-k-1)^{k-r-1}, n-k-2\right)$ with $k-r>2$, or $\lambda=\left((n-k)^{k-2}, n-k-1, r\right)$ with $r<n-k-2$, be a partition for $G(k, n)$. Then the only proper varieties of $G(k, n)$ representing the Schubert class $\sigma_{\lambda}$ are Schubert varieties. In particular, any variety representing $\sigma_{\lambda}$ in $G(k, n)$ is singular.

Proof. Let $X$ be a proper subvariety of $G(k, n)$ representing $\sigma_{(n-k)^{k-2}, n-k-1, r}$. We begin by showing that $X$ is a variety of minimal degree. The dimension of $X$ is $n-k-r+1$. By Pieri's formula, the degree of $X$ in the Plücker embedding is $n-k-r$. By Lemma 4.1, $X$ can be specialized to a Schubert variety. By semi-continuity $([\mathbf{H}]$, III.12.8), the dimension of the linear span of $X$ is at least the dimension of the linear span of a Schubert variety representing the same class. We will next observe that a Schubert variety with class $\sigma_{(n-k)^{k-2}, n-k-1, r}$ is a minimal variety, i.e., it has the maximal possible dimensional linear span given its degree and dimension. (Recall that an irreducible variety of dimension $n$ and degree $d$ spans a linear space of dimension at most $n+d-1([\mathbf{E H}])$.) Since $X$ has the same degree and dimension as the Schubert variety and the dimension of its linear span is at least that of the Schubert variety, we conclude that $X$ must be a minimal variety.

To see that the Schubert variety with class $\sigma_{(n-k)^{k-2}, n-k-1, r}$ is a minimal variety, it suffices to show that the linear span is a projective linear space of dimension at least $2 n-2 k-2 r$. Fix an ordered basis $e_{1}, \ldots, e_{n}$ for the vector space $V$. Let $F_{\bullet}$ be the flag where $F_{i}$ is the span of the first $i$ basis elements $e_{1}, \ldots, e_{i}$. The Schubert variety $\Sigma_{(n-k)^{k-2}, n-k-1, r}\left(F_{\bullet}\right)$ contains the points

$$
p_{1, \ldots, k-2, i, j}=e_{1} \wedge e_{2} \wedge \cdots \wedge e_{k-2} \wedge e_{i} \wedge e_{j},
$$

where $i \in\{k-1, k\}, i<j$ and $j \in\{k, \ldots, n-r\}$. Hence, the span of the Schubert variety in the Plücker embedding contains the span of these Plücker coordinate points. Since there are $2 n-2 k-2 r+1$ such Plücker coordinate points, the projective linear space spanned by the Schubert variety has dimension at least $2 n-2 k-2 r$. Since this is the maximal possible, the dimension of the linear span must equal $2 n-2 k-2 r$. We thus conclude that $X$ is a minimal variety in projective space.

By our assumptions, the degree of $X$ is greater than two. Moreover, the dimension of $X$ is larger than its degree. By the classification of varieties of minimal degree, $X$ must be a cone. In particular, $X$ is singular. Suppose that the vertex of the cone is $p$. (Note that $X$ has a unique singular point. Observe that the Schubert variety with the same class has a unique singular point. By Proposition 4.1 and the semi-continuity of the dimension of the singular locus, the dimension of 
the singular locus of $X$ must be zero. Hence, $X$ is a cone with a unique vertex point.)

By Proposition 3.1, $X$ is contained in a linearly embedded subGrassmannian $G(2, n-k-r+2)$ and has class $\sigma_{n-k-1-r, 0}$ in this sub-Grassmannian. Since $X$ is a cone, the Zariski tangent space of $X$ at $p$ is equal to the linear span of $X$, which is a projective linear space of dimension $2 n-2 k-2 r$. Since the Zariski tangent space to $G(2, n-k-r+2)$ at $p$ also has dimension $2 n-2 k-2 r$ and contains the Zariski tangent space to $X$ at $p$, we conclude that $X \subset Y=$ $T_{p} G(2, n-k-r+2) \cap G(2, n-k-r+2)$. Comparing the Plücker equations of a Schubert variety with class $\sigma_{n-k-1-r, 0}$ with the equations of the Zariski tangent space to $G(2, n-k-r+2)$ at $p$, it is immediate that $Y$ is a Schubert variety with class $\sigma_{n-k-1-r, 0}$. Since both $X$ and $Y$ are irreducible, projective varieties of the same dimension, we conclude that they must be equal. Therefore, $X$ is a Schubert variety. The claim about $\sigma_{(n-k)^{r},(n-k-1)^{k-r-1}, n-k-2}$ follows by the duality between $G(k, n)$ and $G(n-k, n)$. This concludes the proof of the proposition. q.e.d.

Proposition 4.3. Let $\lambda=\left(a^{s}, 0^{k-s}\right) \neq\left(1,0^{k-1}\right)$. Then the only proper subvarieties of $G(k, n)$ representing the cohomology class $\sigma_{\lambda}$ are Schubert varieties. In particular, $\sigma_{\lambda}$ cannot be represented by a smooth, proper subvariety of $G(k, n)$ provided $k>s>0, a \neq n-k$ and $\left(a^{s}, 0^{k-s}\right) \neq\left(1,0^{k-1}\right)$.

Proof. We first prove that if $0<s<k, a \neq n-k$ and $\left(a^{s}, 0^{k-s}\right) \neq$ $\left(1,0^{k-1}\right)$, then $\sigma_{\lambda}$ is singular. It suffices to prove this for the classes $\sigma_{(n-k-1)^{k-1,0}}$ in $G(k, n)$. If $X$ is a smooth variety representing $\sigma_{a^{s}, 0^{k-s}}$, then the intersection of $X$ with a general smooth Schubert variety representing the class $\sigma_{(n-k)^{k-s-1},(n-k-1-a)^{s+1}}$ yields a smooth variety representing the class $\sigma_{(n-k)^{k-s-1},(n-k-1)^{s}, n-k-1-a}$. By Proposition 3.1, this class is supported on a linearly embedded sub-Grassmannian $G(s+$ $1, k+a+1)$ and has the class $\sigma_{(k+a-s-1)^{s}, 0}$ in this sub-Grassmannian. Note that a Schubert variety $\sigma_{(n-k-1)^{k-1,0}}$ in $G(k, n)$ embedded in its Plücker embedding is a cone over the Segre embedding of $\mathbb{P}^{k-1} \times \mathbb{P}^{n-k-1}$. By Lemma 4.1, any variety representing this class is a deformation of the cone over the Segre variety. To conclude the proof, we appeal to the following theorem of Thom, Grauert-Kerner, Schelssinger, KleimanLandolfi:

Rigidity of cones over Segre varieties. [KL, theorem 2.2.8] Let $K$ be a field and let $X=\mathbb{P}_{K}^{n} \times \mathbb{P}_{K}^{m}$ be embedded in projective space by the Segre morphism. If $n \geq 1$ and $m \geq 2$, then the cone over $X$ is rigid over $K$.

By our assumptions, if $k=2$, then $a>1$ and $n-k-1 \geq 2$. Similarly, if $n-k=2$, then $k>2$. We conclude that the assumptions of theorem 2.2.8 of $[\mathbf{K L}]$ are satisfied. Since the cone over the Segre variety admits 
no infinitesimal deformations, $X$ is a cone over a Segre variety and hence is not smooth. Furthermore, the singular point $p_{0}$ of $X$ corresponds to a $k$-plane $\Lambda$. Since $X$ is a cone, for every point $p \in X$, the line joining $p$ and $p_{0}$ is contained in $X$. Since a line in the Grassmannian $G(k, n)$ consists of $k$-planes that contain a fixed $(k-1)$-plane and are contained in a fixed $(k+1)$-plane, we conclude that every $k$-plane corresponding to a point of $X$ must intersect $\Lambda$ in a $(k-1)$-dimensional linear space. Hence, $X$ is contained in the Schubert variety $\Sigma_{(n-k-1)^{k-1}, 0}$. Since they are both irreducible varieties of the same dimension, $X$ equals the Schubert variety $\Sigma_{(n-k-1)^{k-1}, 0}$.

By Corollary 3.2, if $s=0, s=k$, or $a=n-k$, a variety $X$ representing $\sigma_{\lambda}$ is a Schubert variety. We can now prove that if $\left(a^{s}, 0^{k-s}\right) \neq\left(1,0^{k-1}\right)$, then $X$ is a Schubert variety by induction on $n$ and $k-s$. We may assume that $0<a<n-k$ and $0<s<k$. By induction on $k$ and $n$, the singular locus of $X$ has class $\left((a+1)^{s+1}, 0^{k-s-1}\right)$. By induction on $k-s$, the singular locus of $X$ is a Schubert variety of $k$-planes intersecting a fixed linear space $\Lambda_{n-k+s+1-a}$ of dimension $n-k+s+1-a$. Intersect $X$ with a general Schubert variety $\Sigma_{1^{k}}$ defined with respect to a hyperplane $H$. By induction on $n$, the intersection $X \cap \Sigma_{1^{k}}$ is a Schubert variety in $G(k, n-1)$ with class $\left(a^{s}, 0^{k-s}\right)$. Since the singular locus of $X \cap \Sigma_{1^{k}}$ is the Schubert variety of $k$-planes intersecting $\Lambda \cap H$ in a subspace of dimension $n-k+s+1-a$, we conclude that $X \cap \Sigma_{1^{k}}$ is the Schubert variety of $k$-planes intersecting $\Lambda \cap H$ in a subspace of dimension $n-k+s-a$. Since this is true for a general hyperplane $H$, we conclude that the $k$-planes parameterized by $X$ intersect $\Lambda$ in a subspace of dimension $n-k+s-a$. Therefore, $X$ is a Schubert variety. This concludes the proof.

q.e.d.

REMARK 4.4. Example 13 and remark 33 of $[\mathbf{B r}]$ or proposition 3.5 of [Ho2] show that when $\lambda=\left(a^{s}, 0^{k-s}\right)$ with $1<s<k$ and $a>2$, the Schubert classes are Schur rigid.

Proof of Theorem 1.6. We are now ready to prove Theorem 1.6. We need to show that if $\lambda$ is a non-smoothable partition for $G(k, n)$, then $\sigma_{\lambda}$ cannot be represented by a smooth subvariety of $G(k, n)$. Express $\lambda=\left(\mu_{1}^{i_{1}}, \ldots, \mu_{j}^{i_{j}}\right)$, where $\sum i_{l}=k$ and $\mu_{1}>\mu_{2}>\cdots>\mu_{j}$.

We may assume that $\mu_{1} \neq n-k$. Otherwise, by Proposition 3.1, any subvariety of $G(k, n)$ representing $\sigma_{\lambda}$ is contained in a linearly embedded sub-Grassmannian $G\left(k-i_{1}, n-i_{1}\right)$ and represents the class $\left(\mu_{2}^{i_{2}}, \mu_{3}^{i_{3}}, \ldots, \mu_{j}^{i_{j}}\right)$ in this sub-Grassmannian. If the latter class cannot be represented by a smooth subvariety, then $\sigma_{\lambda}$ cannot be represented by a smooth subvariety of $G(k, n)$. Similarly, we may assume that $\mu_{j}=0$. Otherwise, by Proposition 3.1, any subvariety representing $\sigma_{\lambda}$ is contained in a linearly embedded sub-Grassmannian $G\left(k, n-\mu_{j}\right)$ and represents the class $\left(\left(\mu_{1}-\mu_{j}\right)^{i_{1}},\left(\mu_{2}-\mu_{j}\right)^{i_{2}}, \ldots,\left(\mu_{j-1}-\mu_{j}\right)^{i_{j-1}}, 0^{i_{j}}\right)$ 
in this sub-Grassmannian. If the latter class cannot be represented by a smooth subvariety, then $\sigma_{\lambda}$ cannot be represented by a smooth subvariety of $G(k, n)$. Now we proceed by induction on $j$.

- If $j=1$, then $\lambda$ is not non-smoothable and the theorem holds. In fact, the Schubert variety $\Sigma_{\lambda}$ is a linearly embedded sub-Grassmannian and smooth. We may, therefore, assume that $j>1$.

- If $j=2$, since we are assuming that $\mu_{1}<n-k, \lambda$ is non-smoothable if $i_{1}>1$ or if $\mu_{1}>\mu_{2}+1$. In either of these cases, by Proposition 4.3 , any variety representing the class $\sigma_{\lambda}$ is a singular Schubert variety. Note that if $\mu_{1}=n-k$, then $\sigma_{\lambda}$ is the class of a linearly embedded sub-Grassmannian and can be represented by a smooth subvariety of $G(k, n)$. If $i_{1}=1$ and $\mu_{1}=\mu_{2}+1$, then $\sigma_{\lambda}$ is the class of a hyperplane section of a linearly embedded sub-Grassmannian and can be represented by a smooth subvariety of $G(k, n)$.

- If $j=3$, since we are assuming that $\mu_{1}<n-k$, then a non-smoothable partition $\lambda$ satisfies one of the following possibilities.

- If $\mu_{2}>\mu_{3}+1$ or $i_{2}>1$, then applying Case (1) of the Lemma $3.5\left(n-k-\mu_{1}\right)$-times, we conclude that if $\sigma_{\lambda}$ can be represented by a smooth subvariety of $G(k, n)$, then $\sigma_{\lambda^{\prime}}$, with $\lambda^{\prime}=((n-$ $\left.k)^{i_{1}},\left(n-k+\mu_{2}-\mu_{1}\right)^{i_{2}},\left(n-k+\mu_{3}-\mu_{1}\right)^{i_{3}}\right)$, can be represented by a smooth subvariety of $G(k, n)$. Next, applying Case (3) of the Lemma $3.5 i_{1}$-times, we conclude that $\sigma_{\lambda^{\prime}}$ can be represented by a smooth subvariety of $G(k, n)$, then $\sigma_{\lambda^{\prime \prime}}$ with $\lambda^{\prime \prime}=((n-k+$ $\left.\left.\mu_{2}-\mu_{1}\right)^{i_{2}},\left(n-k+\mu_{3}-\mu_{1}\right)^{i_{3}}\right)$ can be represented by a smooth subvariety of $G\left(k-i_{1}, n-i_{1}\right)$. By the case $j=2, \sigma_{\lambda^{\prime \prime}}$ cannot be represented by a smooth subvariety of $G\left(k-i_{1}, n-i_{1}\right)$. Therefore, $\sigma_{\lambda}$ cannot be represented by a smooth subvariety of $G(k, n)$. From now on, we may assume that $\mu_{2}=\mu_{3}+1$ and $i_{2}=1$.

- If $\mu_{1}>\mu_{2}+1$ or $i_{1}>1$, since we are assuming that $\mu_{3}=0$, by applying Cases (2) and (3) of Lemma $3.5\left(i_{3}\right)$-times, we conclude that if $\sigma_{\lambda}$ can be represented by a smooth subvariety of $G(k, n)$, then $\sigma_{\lambda^{\prime}}$ with $\lambda^{\prime}=\left(\mu_{1}^{i_{1}}, \mu_{2}^{i_{2}}\right)$ can be represented by a smooth subvariety of $G\left(k-i_{3}, n-i_{3}\right)$. Since by induction $\sigma_{\lambda^{\prime}}$ cannot be represented by a smooth subvariety of $G\left(k-i_{3}, n-i_{3}\right)$, we conclude that $\sigma_{\lambda}$ cannot be represented by a smooth subvariety of $G(k, n)$.

Note that if $\mu_{1}=n-k, i_{2}=1$ and $\mu_{2}=\mu_{3}+1$, then $\sigma_{\lambda}$ is the class of a hyperplane section of a linearly embedded sub-Grassmannian and can be represented by a smooth subvariety of $G(k, n)$. Similarly, if $i_{1}=i_{2}=i_{3}=1$ and $\mu_{i}=\mu_{i+1}+1$, then $\sigma_{\lambda}$ can be represented by a smooth subvariety of $G(3, n)$. If $\mu_{3}=r$, then $\sigma_{\lambda}$ is the class of the variety parameterizing Schubert cycles $\sigma_{r-3, r-3}$ in the Plücker embedding of $G(2, r)$ that are contained in a general hyperplane section. - Finally, if $j>3$, we can easily reduce to the case $j=3$ by induction. Let $\lambda$ be a non-smoothable partition. Suppose that for $s<j-1, i_{s}>1$ 
or $\mu_{s}>\mu_{s+1}+1$. Then applying Cases (2) and (3) of Lemma $3.5\left(i_{j}\right)$ times, we conclude that if $\sigma_{\lambda}$ can be represented by a smooth subvariety of $G(k, n)$, then $\sigma_{\lambda^{\prime}}$ with $\lambda^{\prime}=\left(\mu_{1}^{i_{1}}, \mu_{2}^{i_{2}}, \ldots, \mu_{j-1}^{i_{j-1}}\right)$ can be represented by a smooth subvariety of $G\left(k-i_{j}, n-i_{j}\right)$. Since by induction on $j$, $\sigma_{\lambda^{\prime}}$ cannot be represented by a smooth subvariety of $G\left(k-i_{j}, n-i_{j}\right)$, we conclude that $\sigma_{\lambda}$ cannot be represented by a smooth subvariety of $G(k, n)$. Similarly, if $i_{j-1}>1$ or $\mu_{j-1}>\mu_{j}+1$, then applying Case (1) of Lemma $3.5\left(n-k-\mu_{1}\right)$-times followed by Case (3) of Lemma $3.5 i_{1}$-times, we conclude that if $\sigma_{\lambda}$ can be represented by a smooth subvariety of $G(k, n)$, then $\sigma_{\lambda^{\prime}}$ with $\lambda^{\prime}=\left(\left(n-k+\mu_{2}-\mu_{1}\right)^{i_{2}}, \ldots,(n-\right.$ $\left.\left.k+\mu_{j-1}-\mu_{1}\right)^{i_{j-1}},\left(n-k+\mu_{j}-\mu_{1}\right)^{i_{j}}\right)$ can be represented by a smooth subvariety of $G\left(k-i_{1}, n-i_{1}\right)$. Since, by induction on $j, \sigma_{\lambda^{\prime}}$ cannot be represented by a smooth subvariety, $\sigma_{\lambda}$ cannot be represented by a smooth subvariety of $G(k, n)$. This concludes the proof of the theorem.

q.e.d.

If $\sigma_{\lambda}$ can be represented by a smooth subvariety $X$ in $G(k, n)$, then, by Theorem 1.6, $\lambda$ has the form $\left((n-k)^{i_{1}}, p, p-1, p-2, \ldots, p-s+\right.$ $\left.1,(p-s)^{i_{s+2}}\right)$. Furthermore, Proposition 3.1 implies that the Schubert class $\sigma_{\lambda^{\prime}}$ with $\lambda^{\prime}=\left(s, s-1, s-2, \ldots, 1,0^{i_{s+2}}\right)$ is also representable by a smooth subvariety of $G\left(k-i_{1}, n-p+s\right)$. Hence, we can restrict our discussion to classes $\sigma_{\lambda}$ with $\lambda=\left(s, s-1, \ldots, 1,0^{l}\right)$ in $G(s+l, n)$. The cup product of the cohomology class $[X]$ of $X$ and the Schubert cycle $\sigma_{\nu}$, with $\nu=\left((n-s-l)^{l+1}, 0^{s-1}\right)$, is zero. We conclude that the incidence correspondence

$$
I=\left\{\left(W_{1}, W_{2}\right) \mid W_{1} \subset W_{2},\left[W_{2}\right] \in X\right\} \subset G(l+1, n) \times X
$$

of pairs of an $(l+1)$-dimensional subspace $W_{1}$ and an $(s+l)$-dimensional subspace $W_{2}$ parameterized by $X$ and containing $W_{1}$ cannot dominate $G(l+1, n)$ under the first projection $\pi_{1}$. On the other hand, considering the cup product of $[X]$ and $\sigma_{\nu^{\prime}}$, with $\nu^{\prime}=\left((n-s-l)^{l},(n-s-l-\right.$ $\left.1), 0^{s-1}\right)$, it is easy to see that the image of $\pi_{1}$ is a divisor in $G(l+1, n)$ with class $\sigma_{1}$. Consequently, $X$ parameterizes Schubert varieties with class $\sigma_{(n-s-l)^{l+1}}$ contained in a hyperplane section of $G(l+1, n)$ in its Plücker embedding. Hence, a Schubert cycle cannot be represented by a smooth subvariety of $G(k, n)$ unless it is the class of a linearly embedded sub-Grassmannian or the class of a variety parameterizing linearlyembedded sub-Grassmannians in a hyperplane section of a Grassmannian. Moreover, as discussed in the Introduction, the classes with $l=1$ can always be represented by smooth subvarieties. Consequently, for Grassmannians $G(k, n)$ with $k=2$ or 3 , Theorem 1.6 can be stated as follows.

Corollary 4.5. Let $\sigma_{\lambda_{1}, \lambda_{2}}$ be a Schubert class in $G(2, n)$. Then the following are equivalent. 
1) $\sigma_{\lambda_{1}, \lambda_{2}}$ can be represented by a smooth subvariety of $G(2, n)$.

2) $\lambda_{1}=n-2$ or $\lambda_{2} \geq \lambda_{1}-1$.

3) $\sigma_{\lambda_{1}, \lambda_{2}}$ is the class of a linearly embedded sub-Grassmannian, or a hyperplane section of a linearly embedded sub-Grassmannian.

Corollary 4.6. Let $\sigma_{\lambda}$ be a Schubert class in $G(3, n)$. Then the following are equivalent.

1) $\sigma_{\lambda}$ can be represented by a smooth subvariety of $G(3, n)$.

2) $\lambda$ is not a non-smoothable partition.

3) $\sigma_{\lambda}$ is the class of a linearly embedded sub-Grassmannian, the hyperplane section of a linearly embedded sub-Grassmannian or the class of planes with class $\sigma_{k-3, k-3}$ contained in a general hyperplane section of the Plücker embedding of $G(2, k)$ for $k \leq n$.

Finally, we prove Theorem 1.3.

Proof of Theorem 1.3. First, observe that the condition for a partition $\lambda=\left(\mu_{1}^{i_{1}}, \ldots, \mu_{j}^{i_{j}}\right)$ to have an index $1 \leq s<j$ with $i_{s}=1$ and $n-k>$ $\mu_{s}=\mu_{s+1}+1$ is invariant under the duality that exchanges Schubert classes in $G(k, n)$ and $G(n-k, n)$.

Let $\lambda=\left(\mu_{1}^{i_{1}}, \ldots, \mu_{j}^{i_{j}}\right)$ be a partition such that there exists an index $1 \leq s<j$ with $i_{s}=1$ and $n-k>\mu_{s}=\mu_{s+1}+1$. We construct a variety representing the cohomology class $\sigma_{\lambda}$, which is not isomorphic to a Schubert variety. The construction is identical to the construction of the variety $X$ in Example 1.4.

First suppose that $s=1$. Let $a_{l}=\sum_{m=1}^{l} i_{m}$. Fix a $(j-1)$-step partial flag $F_{\bullet}$ with vector spaces of dimensions $n-k+a_{i}-\mu_{i}$ for $2 \leq i \leq j$. Fix a hyperplane section $H$ in the Plücker embedding of $G\left(a_{2}, F_{n-k+a_{2}-\mu_{2}}\right)$. Consider the following variety:

$$
\begin{aligned}
Y:= & \left\{[W] \in G(k, n) \mid W^{\prime} \subset W \cap F_{n-k+a_{2}-\mu_{2}} \text { for }\left[W^{\prime}\right] \in H,\right. \\
& \text { and } \left.\operatorname{dim}\left(W \cap F_{n-k+a_{i}-\mu_{i}}\right) \geq a_{i}, 2 \leq i \leq j\right\} .
\end{aligned}
$$

Then $Y$ represents the Schubert class $\sigma_{\lambda}$, but is not a Schubert variety unless the hyperplane section $H$ is a Schubert variety. For example, if $H$ is a smooth hyperplane section, then the singular locus of $Y$ has a different cohomology class than the singular locus of the Schubert variety. $Y$ is not singular along a Schubert variety with class $\sigma_{\left(\mu_{1}+1\right)^{2}, \mu_{2}^{i_{2}-1}, \mu_{3}^{i_{3}}, \ldots, \mu_{j}^{i_{j}}}$.

More generally, suppose $s>1$. Let $F$ be a vector space of dimension $n-k+a_{s+1}-\mu_{s+1}$. Consider the class $\left(\left(\mu_{1}-\mu_{s+1}\right)^{i_{1}}, \ldots,\left(\mu_{s}-\right.\right.$ $\left.\left.\mu_{s+1}\right), 0^{i_{s+1}}\right)$ in $G\left(a_{s+1}, F\right)$. If we consider this class under the isomorphism between $G\left(\operatorname{dim}(F)-a_{s+1}, F\right)$ and $G\left(a_{s+1}, F\right)$, we get a class where $s=1$. Hence, by the previous construction, this class can be represented by a variety $Z$ which is not a Schubert variety. Pick a partial flag

$$
F \subset F_{n-k+a_{s+2}-\mu_{s+2}} \subset \cdots \subset F_{n-k+a_{j}-\mu_{j}} .
$$


The variety $Y$ defined as follows has the class $\left(\mu_{1}^{i_{1}}, \ldots, \mu_{j}^{i_{j}}\right)$, but is not a Schubert variety:

$$
\begin{aligned}
Y:=\{W \in G(k, n) \mid U \subset W \cap F \text { for some } U \in Z \\
\left.\quad \text { and } \operatorname{dim}\left(W \cap F_{n-k+a_{l}-\mu_{l}}\right) \geq a_{l} \text { for } s+1 \leq l \leq j\right\} .
\end{aligned}
$$

This proves that if a Schubert class is rigid, then $\lambda=\left(\mu_{1}^{i_{1}}, \ldots, \mu_{j}^{i_{j}}\right)$ cannot have an index $s$ with $i_{s}=1$ and $n-k>\mu_{s}=\mu_{s+1}+1$.

Suppose that $\lambda=\left(\mu_{1}^{i_{1}}, \ldots, \mu_{j}^{i_{j}}\right)$ is a partition that does not have an index $1 \leq s<j$ such that $i_{s}=1$ and $n-k>\mu_{s}=\mu_{s+1}+1$. Let $X$ be a variety that represents $\sigma_{\lambda}$. We would like to prove that $X$ is a Schubert variety. We will prove this by induction on $j, n$ and the sequence $i_{1}, \ldots, i_{j}$. Define an ordering on sequences $\left(i_{1}, \ldots, i_{j}\right)$ with $\sum i_{s}=k$ by setting $\left(i_{1}^{\prime}, \ldots, i_{l}^{\prime}\right)<\left(i_{1}, \ldots, i_{j}\right)$ either if $l<j$, or if $l=j$, $i_{m}^{\prime}>i_{m}$ for some $m>0$, and $i_{s}^{\prime}=i_{s}$ for every $s<m$. If $j=1$, then by Corollary $3.2, X$ is a Schubert variety.

If $j=2$, then by Proposition $4.3, X$ is a Schubert variety. Suppose that the proposition is true for all $j$ up to $j_{0}$. If $\mu_{1}=n-k$ or $\mu_{j}>0$, by Proposition 3.1, we may assume that $X$ is contained in a linearly embedded sub-Grassmannian. Hence, by induction on $j$ and $n$, we can reduce to the case $\mu_{1}<n-k$ and $\mu_{j}=0$. By intersecting $X$ with a general Schubert variety $\Sigma_{n-k}$ and a general Schubert variety $\Sigma_{1^{k}}$ and induction on $i_{j}$ and $n$, we conclude that the singular locus of $X$ has cohomology class $\sum \sigma_{\lambda_{s}}$, where $\lambda_{s}$ ranges over all singular partitions associated to $\lambda$. In Proposition 4.1, the deformation is given by the group action. Hence, except for the central fiber, the members of the family are isomorphic. In particular, each irreducible component of the singular locus forms a flat family away from the central fiber. The flat limit for each irreducible component has to be contained in the singular locus of the Schubert variety and must be connected in codimension one. Since the singular locus of $X$ and the Schubert variety have the same cohomology class and the components of the singular locus of the Schubert variety in loci of codimension greater than one, we conclude that the singular locus of $X$ must be a union of irreducible components, one for each singular partition associated to $\lambda$.

In particular, the singular locus of $X$ contains a variety $Y$ with class $\sigma_{\lambda^{\prime}}$, where

$$
\lambda^{\prime}=\left(\mu_{1}^{i_{1}}, \ldots,\left(\mu_{t-1}+1\right)^{i_{t-1}+1}, \mu_{t}^{i_{t}-1}, \ldots, \mu_{j}^{i_{j}}\right) .
$$

Since $\mu_{1}<n-k$, we may set $t=2$. Note that $\lambda^{\prime}$ is also a rigid partition unless $i_{2}=2$ and $\mu_{2}=\mu_{3}+1$. Assume first that either $i_{2}>2$ or $\mu_{2}>\mu_{3}+1$. Then, by induction on the sequence $\left(i_{1}, \ldots, i_{j}\right), Y$ is a Schubert variety in $G(k, n)$ defined with respect to a flag

$$
F_{\bullet}=F_{n-k+a_{1}-\mu_{1}} \subset F_{n-k+a_{2}-\mu_{2}} \subset \cdots \subset F_{n-k+a_{j}-\mu_{j}} .
$$


We have to make one exception: if $i_{2}=1$, then $F_{n-k+a_{2}-\mu_{2}}$ should be omitted from the sequence. Let us assume that $i_{2}>1$. Then requiring $k$-planes to intersect $F_{n-k+a_{i}-\mu_{i}}$ in a subspace of dimension $a_{i}$ defines a Schubert variety $\Sigma_{\lambda}$ with the same class as $X$. Intersect the partial flag $F$. with a general codimension one linear space $L$ to obtain a partial flag in $L$. Correspondingly, intersect the variety $X$ with the Schubert variety $\Sigma_{1^{k}}$ defined with respect to $L$. This gives rise to a variety $Z_{L}$ with class $\sigma_{\lambda}$ in $G(k, n-1)$. By induction on $n, Z_{L}$ is a Schubert variety, and it is defined with respect to the partial flag $F_{\bullet} \cap L$. As we vary $L$, the varieties $Z_{L}$ cover $X$ and we conclude that any linear space parameterized by $X$ intersects the linear space $F_{n-k+a_{i}-\mu_{i}}$ in a subspace of dimension at least $a_{i}$. Since both $X$ and $\Sigma_{\lambda}$ are irreducible varieties of the same dimension and $X$ is contained in $\Sigma_{\lambda}$, we conclude that $X=\Sigma_{\lambda}$.

If $i_{2}=1$, the same argument shows that the linear spaces parameterized by $X$ intersect $F_{n-k+a_{i}-\mu_{i}}$ in a subspace of dimension $a_{i}$ with the exception of $i=2$ (which is omitted from the partial flag $F_{\bullet}$ ). If $j>3$, let $R$ be the Zariski closure of the variety $R^{0}$ in $G\left(k-i_{j}, F_{n-k+a_{j-1}-\mu_{j-1}}\right)$ defined by

$$
\begin{aligned}
R^{0}= & \left\{W^{\prime} \in G\left(k-i_{j}, F_{n-k+a_{j-1}-\mu_{j-1}}\right) \mid W^{\prime}=W \cap F_{n-k+a_{j-1}-\mu_{j-1}}\right. \\
& \text { for } \left.W \in X \text { with } \operatorname{dim}\left(W \cap F_{n-k+a_{j-1}-\mu_{j-1}}\right)=k-i_{j}\right\} .
\end{aligned}
$$

Then $R$ has cohomology class $\sigma_{\nu}$ with $\nu=\left(\mu_{1}^{i_{1}}, \ldots, \mu_{j-1}^{i_{j-1}}\right)$ and is a Schubert variety by induction on $j$. It follows that $X$ is also a Schubert variety. If $j=3$, consider the singular locus $Y$ of $X$ with class $\sigma_{\lambda^{\prime}}$, where $\lambda^{\prime}=\left(\mu_{1}^{i_{1}},\left(\mu_{2}+1\right)^{i_{2}+1},\left(\mu_{3}-1\right)^{i_{3}-1}\right)$. By induction on $i_{3}, Y$ is a Schubert variety (even when $i_{1}=1$ and $\mu_{1}=\mu_{2}+2$ since all the $k$ planes are required to intersect $F_{n-k+a_{1}-\mu_{1}}$ in a subspace of dimension $\left.a_{1}\right)$. $Y$ determines the missing partial flag element $F_{n-k+a_{2}-\mu_{2}}$. The argument in the previous paragraph allows us to conclude that $X$ is a Schubert variety.

Finally, suppose $i_{2}=2$ and $\mu_{2}=\mu_{3}+1$. Then let $u<j$ be the smallest index such that $i_{u}>2$ or $\mu_{u}>\mu_{u+1}+1$. If there does not exist such an index, then $i_{s}=2$ and $\mu_{s}=\mu_{s+1}+1$ for all $1<s<j$. Using the duality between $G(k, n)$ and $G(n-k, n)$, we are reduced to the case in the previous paragraph. We may, therefore, assume that there exists such an index $u$. Repeating the argument for the index $t=u$ instead of $t=2$, it is easy to conclude that $X$ is a Schubert variety. This concludes the proof of the theorem.

q.e.d.

\section{References}

[Be] E. Bertini, Introduzione alla geometria proiettiva degli iperspazi, Enrico Spoerri, Pisa, 1907, MR 1548022, JFM 49.0484.08. 
[BL] S. Billey \& V. Lakshmibai, Singular loci of Schubert varieties, Birkhäuser, Boston, MA, 2000, MR 1782635, Zbl 0959.14032.

[Br] R. Bryant, Rigidity and quasi-rigidity of extremal cycles in Hermitian symmetric spaces, Princeton University Press Ann. Math. Studies AM-153, 2005.

[D] Del Pezzo, Sulle superficie di ordine $n$ immerse nello spazio di $n+1$ dimensioni, Nap. rend., XXIV, 1885, 212-216, JFM 19.0841.01.

[EH] D. Eisenbud \& J. Harris, On varieties of minimal degree (a centennial account), Algebraic geometry, Bowdoin 1985, Proc. Sympos. Pure Math. 46 no. 1, Amer. Math. Soc., Providence, RI, 1987, MR 0927946, Zbl 0646.14036.

[F] W. Fulton, Intersection Theory, Springer-Verlag, Berlin Heidelberg, 1998, MR 1644323, Zbl 0885.14002.

[HM] J. Harris \& I. Morrison, Moduli of curves, Springer, New York, 1998, MR 1631825, Zbl 0913.14005.

[H] R. Hartshorne, Algebraic geometry, Springer, 1977, MR 0463157, Zbl 0531.14001.

[Hi] H. Hironaka, A note on algebraic geometry over ground rings. The invariance of Hilbert characteristic functions under the specialization process, Illinois J. Math. 2 (1958), 355-366, MR 0102519, Zbl 0099.15701.

[Ho1] J. Hong, Rigidity of smooth Schubert varieties in Hermitian symmetric spaces, Trans. Amer. Math. Soc. 359 (2007), 2361-2381, MR 2276624, Zbl 1126.14010 .

[Ho2] J. Hong, Rigidity of singular Schubert varieties in $\operatorname{Gr}(m, n)$, J. Differential Geom. 71 no. 1 (2005), 1-22, MR 2191767, Zbl 1137.14311.

[HRT] R. Hartshorne, E. Rees \& E. Thomas, Nonsmoothing of algebraic cycles on Grassmann varieties, Bull. Amer. Math. Soc. 80 no. 5 (1974), 847-851, MR 0357402, Zbl 0289.14011.

[K1] S. Kleiman, Geometry on Grassmannians and applications to splitting bundles and smoothing cycles, Inst. Hautes Études Sci. Publ. Math. 36 (1969), 281297, MR 0265371, Zbl 0208.48501.

[K2] S. Kleiman, The transversality of a general translate, Compos. Math. 28(3) (1974), 287-297, MR 0360616, Zbl 0288.14014.

[KL] S. Kleiman \& J. Landolfi, Geometry and deformation of special Schubert varieties, Compos. Math. 23 no. 4 (1971), 407-434, MR 0314855, Zbl 0238.14006.

[Ko] J. Kollár, Flatness criteria, J. Algebra 175 (1995), 715-727, MR 1339664, Zbl 0853.14008.

[LS] V. Lakshmibai \& C.S. Seshadri, Singular locus of a Schubert variety, Bull. Amer. Math. Soc. vol. 11 no. 2 (1984), 363-366, MR 0752799, Zbl 0549.14016.

[W] M. Walters, Geometry and uniqueness of some extreme subvarieties in complex Grassmannians, Ph. D. thesis, University of Michigan, 1997.

\footnotetext{
UNIVERSity OF ILLINOIS AT CHICAGO Department of Mathematics, Statistics and Computer Science Chicago, IL 60607
}

E-mail address: coskun@math.uic.edu 